JOURNAL OF

SYMPLECTIC GEOMETRY

Volume 9, Number 1, 11-31, 2011

\title{
IMMERSIONS IN A MANIFOLD WITH A PAIR OF SYMPLECTIC FORMS
}

\author{
MahuYa Datta
}

Let $N$ be a manifold with a pair of symplectic forms $\sigma_{1}, \sigma_{2}$, and $M$ a manifold with a pair of closed two-forms $\omega_{1}$ and $\omega_{2}$. For certain pairs of symplectic forms on $N$, we prove the existence of smooth immersions $f: M \rightarrow N$ such that $f^{*} \sigma_{i}=\omega_{i}$ for $i=1,2$.

\section{Introduction}

Let $(N, \sigma)$ be a symplectic manifold with the symplectic form $\sigma$ and $M$ a manifold with a closed two-form $\omega$. An immersion $f: M \rightarrow N$ is said to be a symplectic immersion if $f$ pulls back the form $\sigma$ onto $\omega$. All manifolds and maps in this article are assumed to be smooth. The symplectic immersion theorem of Gromov states that the symplectic immersions $f: M \rightarrow N$ satisfy the $C^{0}$ dense $h$-principle near the continuous maps $f_{0}: M \rightarrow N$ which pull back the deRham cohomology class of $\sigma$ onto that of $\omega[\mathbf{8}, 3.4 .2(\mathrm{~A})]$. The aim of this paper is to generalize this theorem when the manifold $N$ comes equipped with a pair of symplectic forms $\sigma_{1}$ and $\sigma_{2}$ and $M$ has a pair of closed two-forms $\omega_{1}$ and $\omega_{2}$. An immersion $f: M \rightarrow N$ will be called a bisymplectic immersion if it satisfies the relations $f^{*} \sigma_{1}=\omega_{1}$ and $f^{*} \sigma_{2}=\omega_{2}$. The bisymplectic immersions are solutions to a system of first-order partial differential equations (PDEs) on a manifold. In fact, we can associate a first-order partial differential operator $\mathcal{D}$ defined on the space of $C^{\infty}$ maps from $M$ to $N$ such that the bisymplectic maps are solutions to the equation $\mathcal{D}=\left(\omega_{1}, \omega_{2}\right)$ for a given pair of closed two-forms $\omega_{1}, \omega_{2}$ on $M$. This takes us into the theory of $C^{\infty}$ operators.

Generally, to solve a PDE we need to prove an appropriate implicit function theorem so as to obtain a local inversion of the operator $\mathcal{D}$. The implicit function theorem in the present case should ensure the $C^{\infty}$-smoothness (regularity) of the inversions. Gromov proves in $[\mathbf{8}, 2.3]$ that if an $r$ th-order $C^{\infty}$ operator $\mathcal{D}$ is infintesimally invertible on an open subset $\mathcal{U}$ in the space 
of admissible maps defined by a $d$ th-order differential relation for $d \geq r$, then the operator $\mathcal{D}$ restricted to $\mathcal{U}$ is an open map relative to the fine $C^{\infty}$-topologies on the function spaces and there is a smooth local inversion.

In this case, the associated differential operator takes values in closed forms only, so it cannot admit local inversion. However, we observe that there is a first-order differential operator $\overline{\mathcal{D}}$ such that the solutions of the associated PDE give rise to the solutions of the original equation (see Section 4). Moreover, the operator $\overline{\mathcal{D}}$ is infinitesimally invertible on a set of maps which are solutions to some open differential relation. Such maps will be referred as $\left(\sigma_{1}, \sigma_{2}\right)$-regular maps in this paper (see Definition 2.3). We observe in Section 2 that a generic map is $\left(\sigma_{1}, \sigma_{2}\right)$-regular under mild dimension restriction. Applying the implicit function theorem of Gromov we then derive the following result in Section 3.

Theorem A. Let $\sigma_{1}=\sum_{k=1}^{2 q} d x_{k} \wedge d y_{k}$ and $\sigma_{2}=\sum_{k=1}^{q}\left(d x_{2 k-1} \wedge d y_{2 k}-\right.$ $\left.d x_{2 k} \wedge d y_{2 k-1}\right)$ be two linear symplectic forms on $\mathbb{R}^{4 q}$. Let $M$ be a closed manifold with two exact two-forms $\omega_{1}$ and $\omega_{2}$. If $2 q \geq 3 \operatorname{dim} M$ and $q$ is even, then there exists a $\left(\sigma_{1}, \sigma_{2}\right)$-regular bisymplectic immersion $f: M \longrightarrow \mathbb{R}^{4 q}$.

We also partially answer a question of Gromov pertaining to inducing square four-forms from a small perturbation of square symplectic forms. Note that a symplectic immersion $f: M \rightarrow N$ (i.e., $f^{*} \sigma=\omega$ ) satisfies $f^{*}\left(\sigma^{2}\right)=\omega^{2}$. Suppose we break the symmetry of $\sigma^{2}$ by perturbing it a little to $\Omega$. Will it still be possible to induce the form $\omega^{2}$ by an immersion from the perturbed four-form $\Omega ?^{1}$ We take a linear symplectic form $\sigma_{1}$ on $\mathbb{R}^{2 q}$ and some specific perturbation $\sigma_{2}$ of $\sigma_{1}$ which allow $\left(\sigma_{1}, \sigma_{2}\right)$-regular immersions. If we set the wedge of two such forms as $\Omega$ then it is possible to induce the square forms $\omega^{2}$ from $\Omega$ by means of immersions $f: M \rightarrow \mathbb{R}^{2 q}$.

Theorem B. Let $\sigma_{1}=\sum_{k=1}^{2 q} d x_{k} \wedge d y_{k}$ and $\sigma_{2}=\sum_{k=1}^{q} \lambda_{k}\left(d x_{2 k-1} \wedge d y_{2 k-1}+\right.$ $\left.d x_{2 k} \wedge d y_{2 k}\right)$ be two linear symplectic forms on $\mathbb{R}^{4 q}$, where $\lambda_{k}$ 's are distinct real numbers. Then given any exact two-form $\omega$ on a closed manifold $M$, there exists an immersion $f: M \longrightarrow \mathbb{R}^{4 q}$ such that $f^{*}\left(\sigma_{1} \wedge \sigma_{2}\right)=\omega^{2}$ for $2 q \geq 3 \operatorname{dim} M$.

We also prove the following $h$-principle in Section 4.

Theorem C. Suppose $N$ is a smooth manifold with closed two-forms $\sigma_{1}$ and $\sigma_{2}$, and $M$ is an open manifold with a closed two-form $\omega$. Then in the following two cases (a) and (b), the $\left(\sigma_{1}, \sigma_{2}\right)$-regular immersions $f: M \rightarrow N$ which pull back both the forms $\sigma_{1}$ and $\sigma_{2}$ onto $\omega$, satisfy the h-principle in the space of continuous maps $f_{0}: M \rightarrow N$ such that $f_{0}^{*}\left[\sigma_{i}\right]=[\omega]$ for $i=1,2$.

(a) $\omega$ is the zero form on $M$.

(b) $M$ is a symplectic manifold and $\omega$ is a symplectic form on $M$.

\footnotetext{
${ }^{1}$ The question was posed by Gromov in discussions with the author at the IHES in the year 2005 .
} 
The problem of inducing a pair of structures by maps were first considered in $[\mathbf{1}]$ and later in $[\mathbf{2}-\mathbf{4}]$. In all these articles, one of the two structures was a Riemannian metric, while the other structure was a contact form (in [1]), a Riemannian metric (in $[\mathbf{2}, \mathbf{3}]$ ) or a symplectic form (in $[\mathbf{4}]$ ), and except in $[\mathbf{3}]$, the authors exhibit the existence of $C^{1}$-immersions which induce the given pair of structures by adapting Nash's technique [11]. In [3], on the other hand, the authors prove the existence of Lipschitz solutions to the given problem by the convex integration technique $[\mathbf{8}, 2.4]$. In this paper, we consider a pair of symplectic forms and prove the existence of smooth immersions which induce a given pair of closed two-forms. We employ, in contrast with earlier works, the analytic technique and the sheaf technique in the theory of $h$-principle which we discuss in Appendix A.

\section{2. $\left(\sigma_{1}, \sigma_{2}\right)$-Regular maps}

In this section, we introduce the notion of $\left(\sigma_{1}, \sigma_{2}\right)$-regular maps into a manifold $N$ which comes with a pair of closed two-forms $\sigma_{1}$ and $\sigma_{2}$. The main result of this section gives a sufficient condition for the existence of such regular maps when $\sigma_{1}, \sigma_{2}$ is a symplectic pair.

Definition 2.1. Let $\sigma_{1}$ and $\sigma_{2}$ be a pair of linear two-forms on a vector space $W$. A subspace $V$ of $W$ is said to be $\left(\sigma_{1}, \sigma_{2}\right)$-regular (or simply regular) if the linear map

$$
\left(\tilde{\sigma}_{1}, \tilde{\sigma}_{2}\right): W \longrightarrow \Lambda^{1}(V) \times \Lambda^{1}(V)
$$

defined by

$$
\partial \mapsto\left(\partial .\left.\sigma_{1}\right|_{V}, \partial .\left.\sigma_{2}\right|_{V}\right)
$$

is an epimorphism.

A necessary condition for the existence of regular subspaces is that $\operatorname{dim} W \geq 2 \operatorname{dim} V$. If $V$ is a regular subspace of $\left(W, \sigma_{1}, \sigma_{2}\right)$ then any subspace of $V$ is also regular.

Proposition 2.1. $V$ is $\left(\sigma_{1}, \sigma_{2}\right)$-regular if and only if $W=\operatorname{ker} \tilde{\sigma}_{1}+\operatorname{ker} \tilde{\sigma}_{2}$; in other words, ker $\tilde{\sigma}_{1}$ is transversal to ker $\tilde{\sigma}_{2}$.

Proof. Let

$$
\bar{\sigma}_{1}=\left.\tilde{\sigma}_{1}\right|_{\operatorname{ker} \tilde{\sigma}_{2}}: \operatorname{ker} \tilde{\sigma_{2}} \longrightarrow \Lambda^{1}(V) \text { and } \bar{\sigma}_{2}=\left.\tilde{\sigma}_{2}\right|_{\operatorname{ker} \tilde{\sigma_{1}}}: \operatorname{ker} \tilde{\sigma_{1}} \longrightarrow \Lambda^{1}(V) .
$$

Observe that $\operatorname{ker} \bar{\sigma}_{1}=\operatorname{ker} \bar{\sigma}_{2}=\operatorname{ker}\left(\tilde{\sigma}_{1}, \tilde{\sigma}_{2}\right)=\operatorname{ker} \tilde{\sigma}_{1} \cap \operatorname{ker} \tilde{\sigma}_{2}$. If $V$ is regular then both $\bar{\sigma}_{1}$ and $\bar{\sigma}_{2}$ are surjective. The converse is also true. To see this let $\left(\alpha_{1}, \alpha_{2}\right) \in \Lambda^{1}(V) \times \Lambda^{1}(V)$. Then there exist vectors $\partial_{1} \in \operatorname{ker} \tilde{\sigma}_{1}$ and $\partial_{2} \in \operatorname{ker} \tilde{\sigma_{2}}$ such that

$$
\bar{\sigma}_{2}\left(\partial_{1}\right)=\alpha_{2} \text { and } \bar{\sigma}_{1}\left(\partial_{2}\right)=\alpha_{1}
$$


Hence, $\tilde{\sigma}_{1}\left(\partial_{1}+\partial_{2}\right)=\alpha_{1}$ and $\tilde{\sigma}_{2}\left(\partial_{1}+\partial_{2}\right)=\alpha_{2}$ which proves $\left(\sigma_{1}, \sigma_{2}\right)$-regularity of $V$. Consequently, $V$ is regular if and only if the following equalities hold:

$$
\operatorname{dim} \operatorname{ker} \tilde{\sigma}_{1}=\operatorname{dim} V+\operatorname{dim}\left[\operatorname{ker} \tilde{\sigma}_{1} \cap \operatorname{ker} \tilde{\sigma}_{2}\right]=\operatorname{dim} \operatorname{ker} \tilde{\sigma}_{2},
$$

which implies that

$$
\begin{aligned}
\operatorname{dim}\left(\operatorname{ker} \tilde{\sigma}_{1}+\operatorname{ker} \tilde{\sigma}_{2}\right) & =\operatorname{dim} \operatorname{ker} \tilde{\sigma}_{1}+\operatorname{dim} \operatorname{ker} \tilde{\sigma}_{2}-\operatorname{dim}\left[\operatorname{ker} \tilde{\sigma}_{1} \cap \operatorname{ker} \tilde{\sigma}_{2}\right] \\
& =2 \operatorname{dim} V+\operatorname{dim} \operatorname{ker}\left(\tilde{\sigma}_{1}, \tilde{\sigma}_{2}\right) \\
& =\operatorname{dim} W .
\end{aligned}
$$

If $\sigma_{1}$ and $\sigma_{2}$ are two linear symplectic forms on $W$ then we can characterize the regularity condition as follows:

Corollary 2.1. Suppose $\sigma_{1}$ and $\sigma_{2}$ are two linear symplectic forms on $W$. A subspace $V$ is $\left(\sigma_{1}, \sigma_{2}\right)$-regular if and only if

$$
V^{\sigma_{1}} \text { is transversal to } V^{\sigma_{2}} \text {, }
$$

where $V^{\sigma_{i}}=\left\{w \in W: \sigma_{i}(v, w)=0\right.$ for all $\left.v \in V\right\}$ is the symplectic complement of $V$ relative to $\sigma_{i}, i=1,2$.

Proof. We may identify ker $\tilde{\sigma}_{1}$ as the symplectic complement of $V$ relative to $\sigma_{1}$. Similarly ker $\tilde{\sigma}_{2}$ is the symplectic complement of $V$ relative to $\sigma_{2}$.

Proposition 2.2. Let $\sigma_{1}$ and $\sigma_{2}$ be two linear symplectic forms on $W$. Let $A$ denote the unique vector space isomorphism $W \longrightarrow W$ determined by the relation $\sigma_{2}(v, w)=\sigma_{1}(v, A w)$ for all $v, w \in W$. A subspace $V$ of $W$ is $\left(\sigma_{1}, \sigma_{2}\right)$-regular if and only if $V+A(V)$ has the maximum possible dimension.

Proof. We observe that $V^{\sigma_{1}}$ is transversal to $V^{\sigma_{2}}$ if and only if $V^{\sigma_{1}}$ is transversal to $A(V)^{\sigma_{1}}$. Since $(V+A(V))^{\sigma_{1}}=V^{\sigma_{1}} \cap A(V)^{\sigma_{1}}$, we obtain $\operatorname{codim}(V+A(V))^{\sigma_{1}}=2 n$. Consequently, $V+A(V)$ has the maximum possible dimension, as $\sigma_{1}$ is a symplectic form.

Definition 2.2. Let $W$ be a vector space with two linear two-forms $\sigma_{1}, \sigma_{2}$. A linear map $\ell: V \rightarrow W$ from a vector space $V$ to $W$ will be called $\left(\sigma_{1}, \sigma_{2}\right)$ regular if $\ell(V)$ is a regular subspace of $W$. Equivalently, the linear map

$$
\begin{aligned}
W & \rightarrow \Lambda^{1}(V) \times \Lambda^{1}(V), \\
\partial & \mapsto\left(\ell^{*}\left(\partial . \sigma_{1}\right), \ell^{*}\left(\partial . \sigma_{2}\right)\right)
\end{aligned}
$$

is an epimorphism.

Observation. Let $p_{1}$ and $p_{2}$ denote the projection maps of the product vector space $V \times W$ onto $V$ and $W$, respectively. Suppose that $\sigma_{1}, \sigma_{2}$ are two linear two-forms on $W$ and $\omega_{1}, \omega_{2}$ are two linear two-forms on $V$. Let $\bar{\sigma}_{1}=p_{1}^{*} \omega_{1}-p_{2}^{*} \sigma_{1}$ and $\bar{\sigma}_{2}=p_{1}^{*} \omega_{2}-p_{2}^{*} \sigma_{2}$. If $\ell: V \rightarrow W$ is $\left(\sigma_{1}, \sigma_{2}\right)$ regular then the graph map of $\ell, \bar{\ell}: V \rightarrow V \times W$, is $\left(\bar{\sigma}_{1}, \bar{\sigma}_{2}\right)$-regular. This follows from the relation that $\bar{\ell}^{*}\left(\partial . \bar{\sigma}_{i}\right)=\ell^{*}\left(\partial . \sigma_{i}\right)$ for all $\partial \in W, i=1,2$. 
Definition 2.3. Let $\sigma_{1}, \sigma_{2}$ be two closed two-forms on a manifold $N$. A smooth immersion $f: M \longrightarrow N$ is $\left(\sigma_{1}, \sigma_{2}\right)$-regular if the derivative map $d f_{x}: T_{x} M \rightarrow T_{f(x)} N$ is $\left(\sigma_{1}(f(x)), \sigma_{2}(f(x))\right)$-regular for each $x \in M$. We shall often refer the $\left(\sigma_{1}, \sigma_{2}\right)$-regular maps as regular maps.

Theorem 2.1. Consider two linear symplectic forms $\sigma_{1}$ and $\sigma_{2}$ on $\mathbb{R}^{2 q}$ which are related by $\sigma_{2}(v, w)=\sigma_{1}(v, A w)$ for some linear isomorphism $A$ of $\mathbb{R}^{2 q}$. Suppose that $k$ is the maximum of the geometric multiplicities of real eigenvalues of $A$. Then generic maps $f: M \rightarrow \mathbb{R}^{2 q}$ are $\left(\sigma_{1}, \sigma_{2}\right)$-regular immersions for $2 q \geq \max \{3 \operatorname{dim} M, 2 \operatorname{dim} M+k\}$.

In particular, if $A$ has no real eigenvalues then generic maps $f: M \rightarrow \mathbb{R}^{2 q}$ are $\left(\sigma_{1}, \sigma_{2}\right)$-regular immersions for $2 q \geq 3 \operatorname{dim} M$.

Proof. Let $\operatorname{dim} M=n$. Let $\Sigma$ be the subset of the Grassmannian manifold $G r_{n}\left(\mathbb{R}^{2 q}\right)$ which consists of all $n$-planes $T$ in $\mathbb{R}^{q}$ such that $T \cap A(T) \neq 0$. Then $\Sigma$ is the union of two sets $\Sigma^{\prime}$ and $\Sigma^{\prime \prime}$ where

(1) $\Sigma^{\prime}$ consists of all $n$-planes $T$ in $\mathbb{R}^{2 q}$ which contains an eigenvector of $A$, where the eigenspaces of $A$ are at most $k$-dimensional,

(2) $\Sigma^{\prime \prime}$ consists of all $n$-planes $T$ in $\mathbb{R}^{2 q}$ which contains a two-dimensional subspace spanned by a pair $\{v, A v\}$ for some $v \in T$.

Since the geometric multiplicities of the eigenvalues of $A$ are at the most $k$, the dimension of $\Sigma^{\prime}$ is less than or equal to $k-1+(n-1)(2 q-n)$. On the other hand, the dimension of $\Sigma^{\prime \prime}$ is less than or equal to $(2 q-1)+(n-2)(2 q-n)$. Therefore, $\operatorname{dim} \Sigma=\max \left(\operatorname{dim} \Sigma^{\prime}, \operatorname{dim} \Sigma^{\prime \prime}\right)$.

Let $\mathcal{R}$ denote the open subset of $J^{1}\left(M, \mathbb{R}^{2 q}\right)$ consisting of one-jet of germs of immersions from $M$ to $\mathbb{R}^{2 q}$ and let $p: \mathcal{R} \longrightarrow G r_{n}\left(\mathbb{R}^{2 q}\right)$ be the canonical projection which maps an one-jet $j_{f}^{1}(x), x \in M$, onto the $n$-dimensional subspace $\operatorname{Im} d f_{x}$ in $\mathbb{R}^{2 q}$. A smooth map $f: M \longrightarrow \mathbb{R}^{2 q}$ is a regular immersion if and only if $p \circ j_{f}^{1}$ misses the set $\Sigma$, if and only if $j_{f}^{1}$ misses the set $p^{-1}(\Sigma)$.

Now, observe that if $2 q \geq \max \{3 n, 2 n+k\}$ then $\operatorname{codim} \Sigma>n$, and hence the same is true for the codimension of $p^{-1}(\Sigma)$, since $p$ is a submersion. Therefore, by an application of the Thom Transversality Theorem $[\mathbf{7}] j_{f}^{1}$ misses $p^{-1} \Sigma$ for a generic $f$. Thus, a generic map $f: M \longrightarrow \mathbb{R}^{2 q}$ is regular if $2 q \geq \max \{3 n, 2 n+k\}$.

Remark 2.1. If $\sigma_{1}$ and $\sigma_{2}$ are two symplectic forms on a manifold $N$, then there is a bundle isomorphism $A: T N \rightarrow T N$ satisfying the following relation: $\sigma_{2}(v, w)=\sigma_{1}(v, A w)$ for all $v, w \in T_{x} N$ and $x \in N$. Suppose that

$$
k=\max _{x \in M}\left\{\text { geometric multiplicities of the real eigenvalues of } A_{x}\right\} .
$$

Then, we can obtain an exact analogue of Theorem 2.1 for maps $f: M \rightarrow$ $\left(N, \sigma_{1}, \sigma_{2}\right)$ with this $k$. 
Let $\sigma_{1}, \sigma_{2}$ be two linear symplectic forms on $W$ and let $A$ satisfy $\sigma_{2}(v, w)=\sigma_{1}(v, A w)$ for all $v, w \in W$. If $\left\{u_{1}, \ldots, u_{q}, v_{1}, \ldots, v_{q}\right\}$ is a canonical symplectic basis for $\sigma_{1}$ then relative to this basis $A$ can be represented by a matrix of the following form:

$$
M(A)=\left(\begin{array}{cc}
B & C \\
D & B^{t}
\end{array}\right)
$$

where $B, C, D$ are $q \times q$ square matrices of which $C$ and $D$ are skewsymmetric. Indeed, writing $\sigma_{1}=\sum_{k=1}^{q} u_{k}^{*} \wedge v_{k}^{*}$ we have $B=\left(\sigma_{2}\left(u_{j}, v_{i}\right)\right)_{i, j}$, $C=\left(\sigma_{2}\left(v_{i}, v_{j}\right)\right)_{i, j}$ and $D=\left(\sigma_{2}\left(u_{i}, u_{j}\right)\right)_{i, j}$.

If $M(A)$ is symmetric then all eigenvalues are real and if $M(A)$ is skewsymmetric then all eigenvalues are purely imaginary. We consider two special cases under the above criteria. The first, when $B$ is symmetric and $C=D=$ 0 , and the second, when $B$ is skew-symmetric and $C=D=0$.

Example 2.1. Let $\sigma_{1}=u_{k}^{*} \wedge v_{k}^{*}$. If $\sigma_{2}=\sum_{k=1}^{q} \lambda_{k} u_{k}^{*} \wedge v_{k}^{*}$, then $M(A)$ is symmetric. If $q=2 n$ is even and $\sigma_{2}=\sum_{k=1}^{n}\left(u_{2 k-1}^{*} \wedge v_{2 k}^{*}-u_{2 k}^{*} \wedge v_{2 k-1}^{*}\right)$, then $M(A)$ is skew-symmetric.

We can now easily deduce the following two corollaries from Theorem 2.1.

Corollary 2.2. Let $\sigma_{1}=\sum_{i=1}^{q} d x_{i} \wedge d y_{i}$ and $\sigma_{2}=\sum_{i=1}^{q} \lambda_{i} d x_{i} \wedge d y_{i}$ be two symplectic forms on $\mathbb{R}^{2 q}$, where the multiplicities of $\lambda_{i}$ 's are less than equal to $k$. Then a generic map $f: M \rightarrow \mathbb{R}^{2 q}$ is a $\left(\sigma_{1}, \sigma_{2}\right)$-regular immersion for $2 q \geq \max \{3 \operatorname{dim} M, 2 \operatorname{dim} M+k\}$.

Corollary 2.3. If $q$ is even, say $q=2 n$ and $\sigma_{1}=\sum_{i=1}^{2 n} d x_{i} \wedge d y_{i}$ and $\sigma_{2}=\sum_{k=1}^{n}\left(d x_{2 k-1} \wedge d y_{2 k}-d x_{2 k} \wedge d y_{2 k-1}\right)$, then generic maps $f: M \rightarrow \mathbb{R}^{2 q}$ are $\left(\sigma_{1}, \sigma_{2}\right)$-regular immersions for $2 q \geq 3 \operatorname{dim} M$.

We end this section by formulating equivalent criteria for the symmetry and the skew-symmetry conditions on $M(A)$. The following is a standard fact from symplectic geometry $[\mathbf{9}]$.

Lemma 2.1. Let $W$ be a vector space with a linear symplectic form $\sigma$ which is invariant under an almost complex structure $J$. Define a bilinear form $g$ on $W$ by

$$
g(u, v)=\sigma(u, J v) \text { for all } u, v \in W
$$

Then

(1) $\sigma(u, v)=g(J u, v)$;

(2) $g$ is a non-degenerate symmetric form;

(3) $g$ is J-invariant.

The triple $(g, J, \sigma)$ is such that given any two of these structures the third structure is obtained uniquely by the relation $g(u, v)=\sigma(u, J v)$. 
Lemma 2.2. Let $\sigma_{1}$ and $\sigma_{2}$ be two linear symplectic forms on $W$. Let $A$ denote the unique vector space isomorphism $W \longrightarrow W$ determined by the relation $\sigma_{2}(v, w)=\sigma_{1}(v, A w)$. Let $J$ be an almost complex structure on $W$ such that $\sigma_{1}$ is invariant under $J$. Define $g_{1}$ by $g_{1}(v, w)=\sigma_{1}(v, J w)$ for $v, w \in W$. Then the following are equivalent:

(1) $\sigma_{2}$ is invariant under $J$.

(2) A commutes with $J$.

(3) $A$ is symmetric relative to the symmetric form $g_{1}$.

Therefore, under any of the above conditions, eigenvalues of $A$ are all real and they have even multiplicities.

Proof. (1) $\Longrightarrow(2)$ : Since both $\sigma_{1}$ and $\sigma_{2}$ are $J$ invariant, $\sigma_{1}(J u, J A v)=$ $\sigma_{1}(u, A v)=\sigma_{2}(u, v)=\sigma_{2}(J u, J v)=\sigma_{1}(J u, A J v)$ for all $v, w$. Now, the nondegeneracy of $\sigma_{1}$ implies that $A J=J A$. Consequently the eigenvalues occur in pairs.

$(1) \Longrightarrow(3): g_{1}(A u, v)=\sigma_{1}(A u, J v)=-\sigma_{1}(J v, A u)=-\sigma_{2}(J v, u)=$ $\sigma_{2}(u, J v)$. Since $\sigma_{2}$ is $J$-invariant, $\sigma_{2}(u, J v)=\sigma_{2}(v, J u)$ and hence we have $g_{1}(A u, v)=g_{1}(u, A v)$.

$(2) \Longrightarrow(1)$ : Since $A$ commutes with $J$ we obtain $\sigma_{2}(J v, J w)=\sigma_{1}(J v$, $A J w)=\sigma_{1}(J v, J A w)$. Further, since $\sigma_{1}$ is $J$-invariant, $\sigma_{1}(J v$, $J A w)=\sigma_{1}(v, A w)=\sigma_{2}(v, w)$. Thus $\sigma_{2}$ is $J$-invariant.

$(3) \Longrightarrow(1)$ : For any $v, w \in W, \sigma_{2}(J v, J w)=\sigma_{1}(J v, A J w)=-g_{1}(v$, $A J w)=-g_{1}(A v, J w)$ (since $A$ is symmetric with respect to $\left.g_{1}\right)=-g_{1}(J w, A v)=-\sigma_{1}(w, A v)=\sigma_{2}(v, w)$.

If $A$ is symmetric with respect to $g_{1}$ then there is a $g_{1}$-orthonormal basis $u_{1}, u_{2}, \ldots, u_{2 n}$ consisting of eigenvectors of $A$. Suppose $A u_{i}=\lambda_{i} u_{i}$ for $i=$ $1,2, \ldots, 2 n$, where $\lambda_{i}$ are real numbers. Consider $u_{1} \in W$. There exists at least one $u_{n_{1}}, n_{1} \neq 1$, such that $\sigma_{2}\left(u_{1}, u_{n_{1}}\right) \neq 0$. Using the relation between $\sigma_{1}$ and $\sigma_{2}$ we obtain that $\lambda_{1}=\lambda_{n_{1}}$. Now, the $g_{1}$-orthogonal complement $W_{1}$ of the span of $u_{1}$ and $u_{n_{1}}$ is spanned by the set $\left\{u_{i} \mid i \neq 1, i \neq n_{1}\right\}$. Hence, we can repeat the above argument for the pair $\left(W_{1},\left.A\right|_{W_{1}}\right)$, where $\operatorname{dim} W_{1}<\operatorname{dim} W$. Consequently, an induction on $\operatorname{dim} W$ proves that the eigenvalues are real and repeated even number of times.

Analogously, we can prove:

Lemma 2.3. Let $\sigma_{1}$ and $\sigma_{2}$ be two linear symplectic forms on $W$ and $A, J$ and $g_{1}$ be defined as in Lemma 2.2. Then the following are equivalent:

(1) $\sigma_{2}(v, J w)=\sigma_{2}(J v, w)$ for all $v, w \in W$; in other words, $J^{*} \sigma_{2}=-\sigma_{2}$.

(2) A anticommutes with $J$, that is, $A J=-J A$.

(3) $A$ is skew-symmetric relative to the symmetric bilinear form $g_{1}$.

Therefore, under any of the above conditions, eigenvalues of $A$ are purely imaginary. 


\section{Existence of immersions inducing a given pair of forms}

In this section we study the existence of bisymplectic immersions in a manifold $\left(N, \sigma_{1}, \sigma_{2}\right)$, where $\sigma_{1}=d \tau_{1}$ and $\sigma_{2}=d \tau_{2}$ are two exact two-forms on $N$. Let $M$ be a manifold with a pair of exact two-forms $\omega_{1}=d \alpha_{1}$ and $\omega_{2}=d \alpha_{2}$. Consider the differential operator

$$
\mathcal{D}: C^{\infty}(M, N) \rightarrow \Omega^{2}(M) \times \Omega^{2}(M)
$$

which takes an $f \in C^{\infty}(M, N)$ onto the pair $\left(f^{*} \sigma_{1}, f^{*} \sigma_{2}\right)$, where $C^{\infty}(M, N)$ denotes the space of smooth maps from $M$ to $N$ and $\Omega^{2}(M)$ denotes the space of two-forms on $M$. The bisymplectic immersions $f: M \rightarrow N$ are solutions to the differential equation $\mathcal{D} f=\left(\omega_{1}, \omega_{2}\right)$ for the given pair of two-forms on $M$. If $f: M \rightarrow N$ is a bisymplectic immersion, then $f^{*} \tau_{1}-\alpha_{1}$ and $f^{*} \tau_{2}-\alpha_{2}$ are closed one-form, and conversely.

Let us now consider the following first-order differential operator:

$$
\tilde{\mathcal{D}}: C^{\infty}(M, N) \times C^{\infty}(M) \times C^{\infty}(M) \longrightarrow \Omega^{1}(M) \times \Omega^{1}(M)
$$

defined by

$$
\left(f, \phi_{1}, \phi_{2}\right) \mapsto\left(f^{*} \tau_{1}+d \phi_{1}, f^{*} \tau_{2}+d \phi_{2}\right),
$$

where $C^{\infty}(M)$ is the space of smooth real valued functions on $M$ and $\Omega^{1}(M)$ is the space of one-forms on $M$. This operator is closely associated with the operator $\mathcal{D}$; indeed, if $\left(f, \phi_{1}, \phi_{2}\right)$ is a solution of the equation $\tilde{\mathcal{D}}=\left(\alpha_{1}, \alpha_{2}\right)$, then clearly $f$ satisfies the equations $f^{*} \sigma_{1}=\omega_{1}$ and $f^{*} \sigma_{2}=\omega_{2}$.

The linearization of $\tilde{\mathcal{D}}$ at $\left(f, \phi_{1}, \phi_{2}\right)$ is an operator

$$
L: \Gamma^{\infty}\left(f^{*} T N\right) \times C^{\infty}(M) \times C^{\infty}(M) \longrightarrow \Omega^{1}(M) \times \Omega^{1}(M),
$$

which is given by

$$
\left(\partial, \psi_{1}, \psi_{2}\right) \mapsto\left(f^{*}\left(\partial . \sigma_{1}+d\left(\partial . \tau_{1}\right)\right)+d \psi_{1}, f^{*}\left(\partial . \sigma_{2}+d\left(\partial . \tau_{2}\right)\right)+d \psi_{2}\right),
$$

where $\partial$ is a vector field on $N$ along $f$, and $\psi_{1}$ and $\psi_{2}$ are smooth functions on $M . L$ is right invertible if we can solve the following system of equations in $\partial, \psi_{1}$ and $\psi_{2}$ for arbitrary one-forms $g_{1}$ and $g_{2}$ on $M$ :

$$
\begin{array}{cl}
f^{*}\left(\partial . \sigma_{1}\right)=g_{1}, & f^{*}\left(\partial . \sigma_{2}\right)=g_{2}, \\
f^{*}\left(\partial . \tau_{1}\right)+\psi_{1}=0, & f^{*}\left(\partial . \tau_{2}\right)+\psi_{2}=0 .
\end{array}
$$

If $f$ is $\left(\sigma_{1}, \sigma_{2}\right)$-regular (see Definition 2.3), then the first two equations can be solved for $\partial$, the value of which is then inserted in the second set of equations to obtain $\psi_{1}$ and $\psi_{2}$. Thus, $L$ is right invertible by a zeroth-order operator $L^{-1}:\left(g_{1}, g_{2}\right) \mapsto\left(\partial, \psi_{1}, \psi_{2}\right)$ when $f$ is $\left(\sigma_{1}, \sigma_{2}\right)$-regular. Hence, $\tilde{\mathcal{D}}$ is infinitesimally invertible on $\left(\sigma_{1}, \sigma_{2}\right)$-regular immersions. Now, the $\left(\sigma_{1}, \sigma_{2}\right)$-regular immersions are solutions to an open differential relation $A \subset J^{1}(M, N)$. Consequently, the set of regular $C^{\infty}$ immersions, $\mathcal{A}$, form an open subspace 
of $C^{\infty}(M, N)$ in the fine $C^{\infty}$-topology. Hence, we obtain the following result by an application of Theorem A.2 (see Section 5).

Proposition 3.1. The restriction of $\tilde{\mathcal{D}}$ to the space of regular immersions is an open map relative to the fine $C^{\infty}$-topologies on the function spaces.

We are now in a position to prove Theorem A.

Proof of Theorem A. Denote the coordinates on $\mathbb{R}^{2 q}$ by $x_{1}, y_{1}, \ldots, x_{q}, y_{q}$. Suppose that $q=2 n$ and let

$$
\bar{\sigma}_{1}=\sum_{k=1}^{2 n} d x_{k} \wedge d y_{k} \text { and } \bar{\sigma}_{2}=\sum_{k=1}^{n}\left(d x_{2 k-1} \wedge d y_{2 k}-d x_{2 k} \wedge d y_{2 k-1}\right),
$$

so that $\sigma_{1}=\bar{\sigma}_{1} \oplus \bar{\sigma}_{1}$ and $\sigma_{2}=\bar{\sigma}_{2} \oplus \bar{\sigma}_{2}$. Take a $\left(\bar{\sigma}_{1}, \bar{\sigma}_{2}\right)$-regular immersion $h: M \longrightarrow \mathbb{R}^{2 q}$; such an $h$ is guaranteed by Corollary 2.3 since $2 q \geq 3 \operatorname{dim} M$. Define $h^{\prime}: M \longrightarrow \mathbb{R}^{2 q}$ by $h^{\prime}(x)=\left(h_{1}(x),-h_{2}(x), \ldots, h_{2 q-1}(x),-h_{2 q}(x)\right)$, and set $\bar{h}=\left(h, h^{\prime}\right): M \rightarrow \mathbb{R}^{4 q}$. Clearly, $\bar{h}$ is $\left(\sigma_{1}, \sigma_{2}\right)$-regular and it pulls back both $\sigma_{1}$ and $\sigma_{2}$ onto the zero form on $M$, that is, $\bar{h}^{*} \sigma_{i}=0$ for $i=1,2$. Since both $\sigma_{1}$ and $\sigma_{2}$ are exact, we can write $\sigma_{1}=d \tau_{1}$ and $\sigma_{2}=d \tau_{2}$ for some one-forms $\tau_{1}$ and $\tau_{2}$ on $\mathbb{R}^{2 q}$, and this implies that $\bar{h}^{*} \tau_{1}$ and $\bar{h}^{*} \tau_{2}$ are closed one-forms on $M$. Therefore, if we define $\tilde{\mathcal{D}}$ as above then its image contains an ordered pair $\left(c_{1}, c_{2}\right)$ of closed one-forms on $M$, where $c_{i}=\bar{h}^{*} \tau_{i}$ for $i=1,2$. Since $M$ is a closed manifold and $\tilde{\mathcal{D}}$ is an open map (by Proposition 3.1), for every pair of one-forms $\left(\alpha_{1}, \alpha_{2}\right)$ on $M$ there exists a scalar $\lambda>0$ such that $\left(c_{1}+\lambda \alpha_{1}, c_{2}+\lambda \alpha_{2}\right)$ also belongs to the image of $\tilde{\mathcal{D}}$. This implies that, we have a triple $\left(\bar{f}, \phi_{1}, \phi_{2}\right)$ such that $\bar{f}^{*} \tau_{1}+d \phi_{1}=c_{1}+\lambda \alpha_{1}$ and $\bar{f}^{*} \tau_{2}+d \phi_{2}=c_{2}+\lambda \alpha_{2}$, where $\bar{f}: M \rightarrow \mathbb{R}^{4 q}$ is a regular immersion and $\phi_{1}$ and $\phi_{2}$ are smooth functions on $M$. Consequently, $\bar{f}^{*}\left(\sigma_{1}\right)=\lambda d \alpha_{1}$ and $\bar{f}^{*}\left(\sigma_{2}\right)=\lambda d \alpha_{2}$. The required map $f$ is then defined as $f=\lambda^{-\frac{1}{2}} \bar{f}$.

Remark 3.1. Let $\left(N, \sigma_{1}, \sigma_{2}\right)$ and $\left(M, \omega_{1}, \omega_{2}\right)$ be as described in the beginning of this section. Now, suppose that

- the pair $\left(\sigma_{1}, \sigma_{2}\right)$ admits regular immersions $M \rightarrow N$ and

- there exists a diffeomorphism $\phi$ of $N$ such that $\phi^{*} \sigma_{i}=-\sigma_{i}$ for $i=1,2$.

If $M$ is a closed manifold, then by setting $h^{\prime}=\phi \circ h$ it may be seen as in the proof of Theorem A that there exists a regular immersion $f: M \rightarrow N \times N$ which satisfies $f^{*}\left(\sigma_{1} \oplus \sigma_{1}\right)=\omega_{1}$ and $f^{*}\left(\sigma_{2} \oplus \sigma_{2}\right)=\omega_{2}$.

Also, if we have

- a pair of closed two-forms $\sigma_{1}$ and $\sigma_{2}$ on $N$ and

- a $\left(\sigma_{1}, \sigma_{2}\right)$-regular embedding $f: M \rightarrow N$ such that $f^{*} \sigma_{1}=0=f^{*} \sigma_{2}$

then both $\sigma_{1}$ and $\sigma_{2}$ are exact on a tubular neighbourhood of image $f$ in $N$. Therefore, as in the above theorem, an arbitrary pair of exact forms on $M$ can be induced from the pair $\left(\sigma_{1}, \sigma_{2}\right)$ by a regular immersion $\bar{f}$. Moreover, we can choose $\bar{f}$ sufficiently $C^{0}$ close to $f$. 
The next result follows from the above remark, together with Corollary 2.2.

Corollary 3.1. Let

$$
\sigma_{1}=\sum_{k=1}^{2 q} d x_{k} \wedge d y_{k} \text { and } \sigma_{2}=\sum_{k=1}^{q} \lambda_{k}\left(d x_{2 k-1} \wedge d y_{2 k-1}+d x_{2 k} \wedge d y_{2 k}\right)
$$

be two symplectic forms on $\mathbb{R}^{4 q}$, where the multiplicity of each $\lambda_{k}$ is less than or equal to $k$. If $M$ is a closed manifold, then for $2 q \geq \max \{3 \operatorname{dim} M, 2 \operatorname{dim} M+$ $k\}$, there exists a smooth regular immersion $f: M \longrightarrow \mathbb{R}^{4 q}$ such that $f^{*}\left(\sigma_{1}\right)=\omega_{1}$ and $f^{*}\left(\sigma_{2}\right)=\omega_{2}$, where $\omega_{1}$ and $\omega_{2}$ are given exact two-forms on $M$.

Theorem B is now immediate from the above corollary if we take $\omega_{1}=$ $\omega_{2}=\omega$.

We end this section with the following result in $h$-principle.

Theorem 3.1. Let $\sigma_{1}, \sigma_{2}$ be two exact two-forms on a manifold $N$, and $\omega_{1}$ and $\omega_{2}$ two exact two-forms on $M$. Then $\left(\sigma_{1}, \sigma_{2}\right)$-regular maps $f: M \times \mathbb{R} \longrightarrow$ $N$ which pull back the forms $\sigma_{1}$ and $\sigma_{2}$ onto $p^{*} \omega_{1}$ and $p^{*} \omega_{2}$, respectively, satisfy the h-principle.

We postpone the proof of this theorem as of now.

\section{4. $h$-Principle of immersions inducing given pair of forms}

In this section, we assume that $\sigma_{1}$ and $\sigma_{2}$ are arbitrary closed two-forms on $N$ and $\omega_{1}, \omega_{2}$ are two closed two-forms on $M$. We aim to see if the regular maps $f: M \rightarrow N$ satisfying $f^{*} \sigma_{i}=\omega_{i}, i=1,2$ follow the $h$-principle. We first note that such an $f$ pulls back the deRham cohomology classes of $\sigma_{1}$ and $\sigma_{2}$, respectively, onto those of $\omega_{1}$ and $\omega_{2}$. Therefore, the $h$-principle can at most be $C^{0}$-dense (Definition A.2)in the space of continuous maps $f_{0}: M \rightarrow N$ such that $f_{0}^{*}\left[\sigma_{i}\right]=\left[\omega_{i}\right]$ for $i=1,2$, in which case the solution space, if non-empty, is dense in the space of such continuous maps $f_{0}$. In view of this we start with a smooth map $f_{0}: M \rightarrow N$ which satisfies these cohomology conditions. Let $p_{1}$ and $p_{2}$ denote the projection maps of the product manifold $M \times N$ onto $M$ and $N$, respectively. Consider the two product forms on $M \times N$ :

$$
\bar{\sigma}_{1}=: p_{1}^{*} \omega_{1}-p_{2}^{*} \sigma_{1} \text { and } \bar{\sigma}_{2}=: p_{1}^{*} \omega_{2}-p_{2}^{*} \sigma_{2} .
$$

Since the graph of $f_{0}$ is an embedded submanifold of the product manifold $M \times N$, the cohomology condition on $f_{0}$ implies that both $\bar{\sigma}_{1}$ and $\bar{\sigma}_{2}$ are exact in a tubular neighbourhood $Y$ of graph $f_{0}$. Suppose, $\bar{\sigma}_{i}=d \tau_{i}$ for some one-forms $\tau_{i}$ on $Y, i=1,2$. 
If $\bar{f}: M \rightarrow M \times N$ is a section of $p_{1}$ then we will denote the underlying map of $\bar{f}$, namely $p_{2} \circ \bar{f}$, by $f$. If $f$ is $\left(\sigma_{1}, \sigma_{2}\right)$-regular then $\bar{f}$ is $\left(\bar{\sigma}_{1}, \bar{\sigma}_{2}\right)$-regular (see Observation in Section 2).

Let $\Gamma^{\infty}(Y)$ denote the sheaf of $C^{\infty}$ sections of the product bundle $M \times$ $N \rightarrow M$ whose images lie in $Y$. Define a differential operator $\overline{\mathcal{D}}$ as follows:

$$
\begin{aligned}
\Gamma^{\infty}(Y) \times C^{\infty}(M) \times C^{\infty}(M) & \stackrel{\overline{\mathcal{D}}}{\longrightarrow} \Omega^{1}(M) \times \Omega^{1}(M), \\
\left(\bar{f}, \phi_{1}, \phi_{2}\right) & \mapsto\left(\bar{f}^{*} \tau_{1}+d \phi_{1}, \bar{f}^{*} \tau_{2}+d \phi_{2}\right) .
\end{aligned}
$$

If $\overline{\mathcal{D}}\left(\bar{f}, \phi_{1}, \phi_{2}\right)=0$ then $f^{*} \sigma_{1}=\omega_{1}$ and $f^{*} \sigma_{2}=\omega_{2}$.

Now, as we observed in the previous section, $\overline{\mathcal{D}}$ is infinitesimally invertible at all triples $\left(\bar{f}, \phi_{1}, \phi_{2}\right)$ for which $p_{2} \circ \bar{f}$ is $\left(\sigma_{1}, \sigma_{2}\right)$-regular. Consequently, $\overline{\mathcal{D}}$ is a first-order differential operator which admits a zeroth-order inversion of defect 1 (see Section 5).

Let $E$ denote the fibre bundle over $M$ whose total space is $Y \times \mathbb{R} \times \mathbb{R}$ and the projection map $\pi: E \rightarrow M$ is defined by $\pi(y, t, s)=p_{1}(y)$, where $(y, t, s) \in Y \times \mathbb{R} \times \mathbb{R}$. The sections of $E$ are in one-to-one correspondence with triples $\left(\bar{f}, \phi_{1}, \phi_{2}\right)$, where $\bar{f} \in \Gamma^{\infty}(Y)$ and $\phi_{1}, \phi_{2} \in C^{\infty}(M)$. Let $G$ denote the vector bundle $\Lambda^{1}(M) \oplus \Lambda^{1}(M)$, where $\Lambda^{1}(M)$ is the cotangent bundle of $M$. The operator $\overline{\mathcal{D}}$ induces a sequence of bundle maps $\bar{\Delta}_{\alpha}: E^{(\alpha+1)} \rightarrow G^{(\alpha)}$, $\alpha \geq 0$, satisfying the relations $\bar{\Delta}_{\alpha} \circ j_{\left(\bar{f}, \phi_{1}, \phi_{2}\right)}^{\alpha+1}=j_{\overline{\mathcal{D}}\left(\bar{f}, \phi_{1}, \phi_{2}\right)}^{\alpha}$ (see Section 5). For each non-negative integer $\alpha$, we now define a differential relation $\overline{\mathcal{R}}^{\alpha}=$ $\bar{\Delta}_{\alpha}^{-1}(0)$. All these relations have the same $C^{\infty}$ solutions as the equation $\overline{\mathcal{D}}=0$.

Let $\overline{\mathcal{R}}_{2}$ be the subset of $\overline{\mathcal{R}}^{2}$ consisting of all three-jets at $x, x \in M$, which can be represented by a local section $\left(\bar{f}, \phi_{1}, \phi_{2}\right)$ of $E$ such that $p_{2} \circ \bar{f}$ is regular at $x$. Therefore, $\left(\bar{f}, \phi_{1}, \phi_{2}\right)$ is a solution of $\overline{\mathcal{R}}_{2}$ if

(1) $f=p_{2} \circ \bar{f}$ is $\left(\sigma_{1}, \sigma_{2}\right)$-regular, and

(2) $\bar{f}^{*} \tau_{1}+d \phi_{1}=0$ and $\bar{f}^{*} \tau_{2}+d \phi_{2}=0$, that is $\overline{\mathcal{D}}\left(\bar{f}, \phi_{1}, \phi_{2}\right)=0$.

Let $\bar{\Phi}$ denote the solution sheaf of $\overline{\mathcal{R}}_{2}$ and $\bar{\Psi}_{2}$ the sheaf of sections of $\overline{\mathcal{R}}_{2}$.

The next result follows from Theorem A.2 and Proposition A.1 in Section 5 .

Proposition 4.1. $\bar{\Phi}$ is a microflexible sheaf. Moreover, the three-jet map $j^{3}: \bar{\Phi} \rightarrow \bar{\Psi}_{2}$ is a local weak homotopy equivalence; in other words, $\overline{\mathcal{R}}_{2}$ satisfies the local parametric h-principle.

Remark 4.1. Let $\Phi$ denote the sheaf of regular solutions of the original differential equation, namely, $\mathcal{D} f \equiv\left(f^{*} \sigma_{1}, f^{*} \sigma_{2}\right)=\left(\omega_{1}, \omega_{2}\right)$. Let $\mathcal{R}_{1}$ be the subset of $J^{2}(M, N)$ consisting of two-jets of infinitesimal regular solutions of order 1 of the differential equation $\mathcal{D}=\left(\omega_{1}, \omega_{2}\right)$ and $\Psi_{1}$ the sheaf of sections of $\mathcal{R}_{1}$. There is a canonical map $p^{\prime}: \bar{\Phi} \rightarrow \Phi$ which takes the triple $\left(\bar{f}, \phi_{1}, \phi_{2}\right)$ onto $p_{2} \circ \bar{f}$. Then $p^{\prime}$ induces a map $p: \overline{\mathcal{R}}_{2} \rightarrow \mathcal{R}_{1}$ defined by $\left(j_{\bar{f}}^{3}, j_{\phi_{1}}^{3}, j_{\phi_{2}}^{3}\right)(x) \mapsto j_{p_{2} \circ \bar{f}}^{2}(x)$. To see this, we note that the exterior differential 
operator $d$ determines, for each $k \geq 1$, a sequence of bundle maps $d_{\alpha}$ : $\left(\Lambda^{k}(M)\right)^{(\alpha+1)} \longrightarrow\left(\Lambda^{k+1}(M)\right)^{(\alpha)}, \alpha=0,1,2, \ldots$ such that $d_{\alpha} \circ j_{\tau}^{\alpha+1}=j_{d \tau}^{\alpha}$, where $\tau$ is a $k$-form. Therefore, if $j_{\left(\bar{f}^{*} \tau_{1}+d \phi_{1}\right)}^{2}=0$ and $j_{\left(\bar{f}^{*} \tau_{2}+d \phi_{2}\right)}^{2}=0$ at $x$, then applying $d_{1}$ on both sides we get $j_{f^{*} \sigma_{1}}^{1}(x)=j_{\omega_{1}}^{1}(x)$ and $j_{f^{*} \sigma_{2}}^{1}(x)=$ $j_{\omega_{2}}^{1}(x)$, where $f=p_{2} \circ \bar{f}$. Thus, $f$ is an infinitesimal solution of order 1 of the equation $\mathcal{D}=\left(\omega_{1}, \omega_{2}\right)$.

Further, we have the following commutative diagram which relates the solution sheaves of the two differential equations:

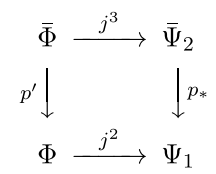

where $p_{*}$ is the map induced by $p$. It can be proved following [5] that $p$ : $\overline{\mathcal{R}}_{2} \rightarrow \mathcal{R}_{1}$ is a surjective submersion and the fibres of $p$ are affine subspaces; hence $p$ has a section. These are, in fact, consequences of the following sequence of vector bundles and maps which is exact by the formal Poincaré Lemma:

$$
\cdots \longrightarrow\left(\Lambda^{k-2}(M)\right)^{(3)} \stackrel{d_{2}}{\longrightarrow}\left(\Lambda^{k-1}(M)\right)^{(2)} \stackrel{d_{1}}{\longrightarrow}\left(\Lambda^{k}(M)\right)^{(1)} \longrightarrow \cdots .
$$

Since $p$ has a section, $p_{*}$ is onto. It is now easy to see from the above commutative square, that if $\overline{\mathcal{R}}_{2}$ satisfies the $h$-principle, then $\mathcal{R}_{1}$ also satisfies the $h$-principle.

We recall the following definitions from $[8,3.4 .1(\mathrm{~B})]$.

Definition 4.1. Let $M$ be a smooth manifold with a closed two-form $\omega$. A vector field $\partial$ on $M$ is said to be $\omega$-isometric if the Lie derivative $L_{\partial} \omega=0$, in other words, $\partial . \omega$ is a closed form. The vector field $\partial$ is said to be $\omega$-exact if there exists a zero-form $\alpha$ (i.e., a function on $M$ ) such that $\partial . \omega=d \alpha$.

A (local) isotopy $\delta_{t}: U \rightarrow M$ is called exact if $\delta_{t}^{\prime}=\frac{d \delta_{t}}{d t}$ is an exact vector field on $\delta_{t}(U)$ for all $t \in[0,1]$ and there exists a homotopy of zero-forms $\alpha_{t}$ defined on $\delta_{t}(U)$ such that $\delta_{t}^{\prime} \cdot \omega=d \alpha_{t}$.

Observation. The isotopy defined by a $\omega$-isometric vector field consists of diffeomorphisms which preserve the form $\omega$. If $\delta_{t}$ is a $\omega$ exact diffeotopy which fixes an open set $U_{0}$ pointwise then the exact one-forms $\delta_{t}^{\prime} \cdot \omega$ vanish on $U_{0}$. This means that any primitive of $\delta_{t}^{\prime}$. $\omega$ takes a constant real value on $U_{0}$. Hence, we can choose a primitive $\phi_{t}$ which also vanishes on the set $U_{0}$. We would like to remark here that diffeotopies with this property are referred as strictly exact diffeotopy in [8, 3.4.1].

Lemma 4.1. Suppose that $\omega_{1}=\omega_{2}=\omega$. Then the $\omega$-exact diffeotopies of $M$ act on the sheaf $\bar{\Phi}$. 
Proof. We follow $[8,3.4 .1(\mathrm{~B})]$ to define an action of strictly $\omega$-exact diffeotopies on $\bar{\Phi}$. First note that each diffeotopy $\delta_{t}: U \rightarrow V$ of open subsets of $M$ lifts to a diffeotopy $\bar{\delta}_{t}: U \times N \rightarrow V \times N$ by $\bar{\delta}_{t}(u, x)=\left(\delta_{t}(u), x\right)$.

Suppose that, $Y^{\prime} \subset Y \cap(U \times N)$ and $\bar{\delta}_{t}\left(Y^{\prime}\right) \subset Y$ for all $t$. Then $\delta_{t}$ has a natural action on sections $\bar{f}: V \rightarrow V \times N$ whose images lie in $Y$. The action is given by $\delta_{t} \cdot \bar{f}=\bar{\delta}_{t}^{-1} \bar{f} \delta_{t}$ (see Example A.1 in Section 5).

Differentiating the homotopy of one-forms $\bar{\delta}_{t}^{*} \tau_{1}$ with respect to $t$ we obtain

$$
L_{\bar{\delta}_{t}^{\prime}} \tau_{1}=\bar{\delta}_{t}^{\prime} \cdot \bar{\sigma}_{1}+d\left(\bar{\delta}_{t}^{\prime} \cdot \tau_{1}\right)=p_{1}^{*}\left(\delta_{t}^{\prime} \cdot \omega\right)+d\left(\bar{\delta}_{t}^{\prime} \cdot \tau_{1}\right) .
$$

If $\delta_{t}$ is strictly $\omega$-exact, then there exists a homotopy of $C^{\infty}$ functions $\alpha_{t}$ along $\delta_{t}(U)$ such that $\delta_{t}^{\prime} \cdot \omega=d \alpha_{t}$. Hence

$$
\bar{\delta}_{t}^{*} \tau_{1}=\tau_{1}+d \phi_{t}, \text { where } \phi_{t}=\int_{0}^{t}\left(p_{1}^{*} \alpha_{t}+\bar{\delta}_{t}^{\prime} \cdot \tau_{1}\right) d t .
$$

Further, if $\delta_{t}$ is constant on $U_{0}$ for $t \leq t_{0}$, we can and we do choose $\phi_{t}=0$ for $t \leq t_{0}$.

Consider a triple $(\bar{f}, \phi, \psi)$ in $\bar{\Phi}$ so that $\bar{f}: V \rightarrow V \times N$ has its image in $Y$ and $\bar{f}^{*} \tau_{1}+d \phi=0, \bar{f}^{*} \tau_{2}+d \psi=0$. If we define $\delta_{t} \cdot \bar{f}=\bar{\delta}_{t}^{-1} \bar{f} \delta_{t}$, then using equation (4.1) we obtain

$$
\begin{aligned}
\left(\delta_{t} \cdot \bar{f}\right)^{*} \tau_{1} & =\delta_{t}^{*} \bar{f}^{*}\left(\bar{\delta}_{t}^{-1}\right)^{*} \tau_{1} \\
& =\delta_{t}^{*} \bar{f}^{*}\left[\tau_{1}-d\left(\bar{\delta}_{t}^{-1}\right)^{*} \phi_{t}\right] \\
& =-\delta_{t}^{*} d \phi+d\left(\delta_{t} \cdot \bar{f}\right)^{*} \phi_{t} \\
& =-d\left[\delta_{t}^{*} \phi-\left(\delta_{t} \cdot \bar{f}\right)^{*} \phi_{t}\right] .
\end{aligned}
$$

Finally, since $\delta_{t}$ is strictly $\omega$-exact diffeotopy, $\phi \mapsto \delta_{t}^{*} \phi-\left(\delta_{t} \cdot \bar{f}\right)^{*} \phi_{t}$ defines an action on the space of $C^{\infty}$ functions on $M$ (for a fixed $\bar{f}$ ). Indeed, if $\delta_{t}$ is constant in $t$ on a maximal open set $U_{0}$ then we can choose $\phi_{t}=0$ on $U_{0}$ and then $\delta_{t}^{*} \phi-\left(\delta_{t} \cdot \bar{f}\right)^{*} \phi_{t}$ is constant on $U_{0}$.

Therefore, we can define the action of a strictly exact diffeotopy $\delta_{t}$ satisfying $\bar{\delta}_{t}\left(Y^{\prime}\right) \subset Y$ on $\bar{\Phi}$ by

$$
\delta_{t} \cdot(\bar{f}, \phi, \psi)=\left(\delta_{t} \cdot \bar{f}, \delta_{t}^{*} \phi-\left(\delta_{t} \cdot \bar{f}\right)^{*} \phi_{t}, \delta_{t}^{*} \psi-\left(\delta_{t} \cdot \bar{f}\right)^{*} \psi_{t}\right),
$$

where $\delta_{t} \cdot \bar{f}=\bar{\delta}_{t}^{-1} \bar{f} \delta_{t}$ and $\phi_{t}, \psi_{t}$ satisfy the relations $\bar{\delta}_{t}^{*} \tau_{1}=\tau_{1}+d \phi_{t}, \bar{\delta}_{t}^{*} \tau_{2}=$ $\tau_{2}+d \psi_{t}$.

Proposition 4.2. Suppose that $\omega_{1}=\omega_{2}=\omega$, where $\omega$ is the zero-form or a symplectic form on $M$. If $M_{0}$ is a submanifold of $M$ of positive codimension then $j^{3}:\left.\left.\bar{\Phi}\right|_{M_{0}} \rightarrow \bar{\Psi}_{2}\right|_{M_{0}}$ is a weak homotopy equivalence.

Proof. In view of Theorem A.3 in Section 5 and Proposition 4.1 we need to show that there is an appropriate class of (local) diffeotopies which act on the sheaf $\bar{\Phi}$ and have the desired sharply moving property. We first consider 
the case when $\omega$ is a symplectic form. Recall that $\omega$ defines a canonical isomorphism $I_{\omega}: T M \rightarrow T^{*} M$ which in turn defines a one-to-one correspondence between vector fields and one-forms on $M$. Indeed, if $\partial$ is a vector field on $M$ then $\partial . \omega$ is a global one-form on $M$. The $\omega$-exact (local) diffetopies are obtained by integrating the vector fields which correspond to the exact one-forms under this correspondence. As we observed in Lemma 4.1, these diffeotopies act on the sheaf $\bar{\Phi}$. It is also known that these diffeotopies sharply move any submanifold of $M$ of positive codimension (see [8, 3.4.2]). This proves the proposition for the case when $\omega$ is symplectic.

If $\omega$ is the zero form, it is enough to observe that any local diffeotopy is $\omega$-exact.

We are now in a position to prove Theorem C.

Proof of Theorem C. Since $M$ is an open manifold, it admits a Morse function without any critical point of index equal to the dimension of $M[\mathbf{1 0}]$. It then follows from Morse theory that, there is a simplicial complex $K$ in $M$ of positive codimension which is a strong deformation retract of $M$. In fact, $M$ is isotopic to an arbitrarily small open neighbourhood of $K$. Now, by the above proposition, the $h$-principle for $\bar{\Phi}$ localizes near $K$, and hence the $h$-principle for the sheaf $\Phi$ also localizes near $K$ (see Remark 4.1). This means that a section of $\mathcal{R}_{1}$ can be homotoped to a solution $f_{1}$ of the differential equation $\mathcal{D}=(\omega, \omega)$ near $K$, where $\omega$ is either the zero-form or a symplectic form on $M$.

To prove (a), take an isotopy $\phi_{t}$ such that $\phi_{1}$ brings $M$ into the domain of $f_{1}$. Then the composition map $f_{1} \circ \phi_{1}$ is a global solution of the equation $\mathcal{D}=(0,0)$.

To obtain global $h$-principle stated in (b) we observe that there is a homotopy of symplectic immersions $\phi_{t}:(M, \omega) \rightarrow(M, \omega)$ such that $\phi_{0}=$ id and $\phi_{1}$ maps $M$ into $K([\mathbf{5}, \mathbf{6}])$. Composing $f_{1}$ with $\phi_{1}$ we obtain a global solution of the equation $\mathcal{D}=(\omega, \omega)$.

Remark 4.2. If $\left(M_{0}, \omega_{0}\right)$ is a symplectic manifold then as a direct consequence of the above theorem we obtain the $h$-principle with $M=M_{0} \times \mathbb{R}^{2}$ and $\omega=\omega_{0} \oplus d x \wedge d y$.

Proof of Theorem 3.1. Let $\omega_{1}=d \alpha_{1}$ and $\omega_{2}=d \alpha_{2}$ for some one-forms $\alpha_{1}, \alpha_{2}$ on $M$. Let $\tilde{\Phi}$ denote the sheaf of solutions of the differential equation $\tilde{\mathcal{D}}=\left(p^{*} \alpha_{1}, p^{*} \alpha_{2}\right)$, where $\tilde{\mathcal{D}}$ is defined as in Section 3. A diffeomorphism $\lambda: M \times \mathbb{R} \rightarrow M \times \mathbb{R}$ is said to be fibre-preserving if $p \circ \lambda=\lambda$, where $p: M \times \mathbb{R} \rightarrow M$ is the projection onto the first factor. Hence $\lambda^{*} p^{*} \alpha_{i}=p^{*} \alpha_{i}, i=1,2$, for such a $\lambda$. This allows us to define an action of fibre-preserving diffeomorphisms on the sheaf $\tilde{\Phi}$. Indeed, if $\tilde{\mathcal{D}}\left(f, \phi_{1}, \phi_{2}\right)=$ $\left(p^{*} \alpha_{1}, p^{*} \alpha_{2}\right)$ and $f$ is regular, then $f^{*} \tau_{i}+d \phi_{i}=p^{*} \alpha_{i}$, where $\sigma_{i}=d \tau_{i}$ for $i=1,2$. If $\lambda$ is a fibre-preserving diffeomorphism then we define an action 
by the following simple rule:

$$
\lambda .\left(f, \phi_{1}, \phi_{2}\right)=\left(f \circ \lambda, \phi_{1} \circ \lambda, \phi_{2} \circ \lambda\right) .
$$

(Note that if $\lambda_{t}$ is a fibre-preserving diffeotopy, then the vector fields $\lambda_{t}^{\prime}$ has no component along $M_{0}$. Hence, $\lambda_{t}^{\prime}$ is $\omega$-exact for any two-form $\omega$ of the form $p_{1}^{*} \omega_{0}$, where $\omega_{0}$ is a two-form on $M$.) Since we observed in Proposition 3.1 that $\tilde{\mathcal{D}}$ is infinitesimally invertible on regular maps, the hypothesis of Theorem A.4 is satisfied (by Theorem A.2 and Proposition A.1 in Section 5). This proves that $\tilde{\Phi}$ satisfies the $h$-principle. This $h$-principle then descends to the desired $h$-principle for $\Phi$ by an argument similar to that in Remark 4.1.

\section{Appendix A. Preliminaries of $\boldsymbol{h}$-principle}

Here we briefly discuss the sheaf technique and the analytic technique in the theory of $h$-principle following [8].

Let $p: E \longrightarrow M$ be a $C^{\infty}$-fibration, and let $E^{(r)}$ denote the $r$-jet space of $C^{\infty}$-sections of $E$ for $r \geq 1$. Then the canonical projection $p^{(r)}: E^{(r)} \longrightarrow M$ is also a fibration. We endow the space of sections of $p$ and $p^{(r)}$ with the $C^{\infty}$ and $C^{0}$-compact open topologies, respectively. The canonical projection maps $E^{(r)} \rightarrow E^{(i)}$ are denoted by $p_{i}^{r}$.

A partial differential relation of order $r$ for sections of $E$ is a subset $\mathcal{R}$ of $E^{(r)}$. A section $f: M \longrightarrow E$ is said to be a solution of $\mathcal{R}$ if the $r$-jet map $j_{f}^{r}$ (which is a section of $p^{(r)}$ ) maps $M$ into $\mathcal{R}$. A section of $p^{(r)}$ is called holonomic if it is the $r$-jet map of a solution of $\mathcal{R}$.

We denote the space of solutions of $\mathcal{R}$ by $\operatorname{Sol} \mathcal{R}$, while $\Gamma(\mathcal{R})$ denotes the space of sections of $E^{(r)} \longrightarrow M$ whose images lie in $\mathcal{R}$.

Definition A.1. A relation $\mathcal{R}$ is said to satisfy the $h$-principle if a section of $\mathcal{R}$ can be homotoped within $\Gamma(\mathcal{R})$ to a holonomic section.

$\mathcal{R}$ satisfies the parametric h-principle if the $r$-jet map $j^{r}: \operatorname{Sol} \mathcal{R} \longrightarrow \Gamma(\mathcal{R})$ is a weak homotopy equivalence.

Definition A.2. Let $\mathcal{S}$ be a subspace of the space of continuous sections of $E$. A relation $\mathcal{R} \subset E^{(r)}$ is said to satisfy the $h$-principle $C^{0}$-dense in $\mathcal{S}$ if for every $f_{0} \in \mathcal{S}$, for every neighbourhood $U$ of graph $f_{0}$ and for every section $\phi_{0}: M \rightarrow \mathcal{R}$ satisfying $p_{0}^{r} \circ \phi_{0}=j_{f_{0}}^{0}$, there exists a homotopy of sections $\phi_{t}: M \rightarrow \mathcal{R}$ such that the image of $p_{0}^{r} \circ \phi_{t}$ is contained in $U$ and $\phi_{1}$ is holonomic.

Let $\Phi$ denote the sheaf of solutions of a given relation $\mathcal{R}$, and $\Psi$ the sheaf of sections of $\mathcal{R}$. The topologies on $\Phi(U)$ and $\Psi(U)$ are, respectively, the $C^{\infty}$ and $C^{0}$ compact open topologies. The $r$-jet map $j^{r}$ defines a sheaf homomorphism from $\Phi$ to $\Psi$. This takes us into the realm of topological sheaves. 
Sometimes, by an abuse of language, we say that the sheaf $\Phi$ satisfies the $h$-principle without giving any reference to a partial differential relation. But one should be careful at this point, since two subsets $\mathcal{R}$ and $\mathcal{R}^{\prime}$ possibly in different jet spaces $E^{(r)}$ and $E^{(s)}$ may have the same set of solutions, but one of the relations may satisfy the $h$-principle while the other may not. In fact, given an $r$ th-order relation $\mathcal{R}$, we can form an $s$ th-order relation $\mathcal{R}^{\prime}$ (for any $s>r$ ) by taking $s$-jets of $C^{s}$ solutions of $\mathcal{R}$.

We recall some general definitions and terminology from $[\mathbf{8}]$.

Definition A.3. Let $\mathcal{F}$ be a topological sheaf over $M$ and $A$ a compact set in $M$. Then $\mathcal{F}(A)$ will denote the direct limit of the sets $\mathcal{F}(U)$, where $U$ runs over all open sets containing $A$.

However, these sets, $\mathcal{F}(A)$, will have only quasi-topological structures [8, 1.4.1]. A map $f: P \longrightarrow \mathcal{F}(A)$ on a polyhedron $P$ is called continuous (in the quasi-topological sense) if there exists an open set $U \supset A$ such that each $f_{p}$ is defined over $U$ and the resulting map $P \longrightarrow \mathcal{F}(U)$ is continuous with respect to the given topology on $\mathcal{F}(U)$.

Definition A.4. $\mathcal{R}$ satisfies the local parametric $h$-principle if for each $x \in$ $M, j^{r}: \Phi(x) \longrightarrow \Psi(x)$ is a weak homotopy equivalence.

Definition A.5. A topological sheaf $\mathcal{F}$ over $M$ is flexible if the restriction maps $\mathcal{F}(A) \longrightarrow \mathcal{F}(B)$ are Serre fibrations for every pair of compact sets $(A, B), A \supset B$. The restriction map $\mathcal{F}(A) \longrightarrow \mathcal{F}(B)$ is called a microfibration if given a continuous map $f_{0}^{\prime}: P \longrightarrow \mathcal{F}(A)$ on a polyhedron $P$ and a homotopy $f_{t}, 0 \leq t \leq 1$, of $\left.f_{0}^{\prime}\right|_{B}$ there exists an $\varepsilon>0$ and a homotopy $f_{t}^{\prime}$ of $f_{0}^{\prime}$ such that $f_{t}^{\prime} \mid \mathrm{Op} B=f_{t}$ for $0 \leq t \leq \varepsilon$. If for every pair of compact sets the restriction morphism is a microfibration, then the sheaf $\mathcal{F}$ is called microflexible.

The following topological result provides a sufficient condition for a sheaf homomorphism to be a weak homotopy equivalence.

Theorem A.1 $([8,2.2 .1(\mathrm{~B})])$. Let $\mathcal{F}$ and $\mathcal{G}$ be two flexible sheaves over $M$, and let $\alpha: \mathcal{F} \longrightarrow \mathcal{G}$ be a continuous sheaf homomorphism such that $\alpha(x): \mathcal{F}(x) \longrightarrow \mathcal{G}(x)$ is a weak homotopy equivalence for each $x \in M$. Then $\alpha$ is a weak homotopy equivalence.

Thus, if the solution sheaf $\Phi$ is flexible and if $\mathcal{R}$ satisfies the local parametric $h$-principle, then $\mathcal{R}$ satisfies the parametric $h$-principle (because $\Psi$ is always flexible $\left.\left[\mathbf{8}, 1.4 .2\left(\mathrm{~A}^{\prime}\right)\right]\right)$.

The solution sheaf turns out to be non-flexible in many important problems, though microflexibility is a much more common property. For example, when $\mathcal{R}$ is open the solution sheaf is easily seen to be microflexible; however, many of the relations that are of special interest are fibrewise closed in the 
jet space. This is the case, when the solutions of $\mathcal{R}$ also arise as solutions to some PDEs $\mathcal{D}(f)=g$, where $\mathcal{D}$ is defined on sections of the fibre bundle $E$ taking values in the space of sections of a vector bundle. Gromov proves that $\Phi$ (or possibly a subsheaf of $\Phi$ ) is microflexible when the operator $\mathcal{D}$ is infinitesimally invertible over an open subset of $\Gamma^{\infty}(E)$ in the fine $C^{\infty}$ topology. On the other hand, he observes that there are higher order relations $\left.\mathcal{R}^{\alpha} \subset J^{(r+\alpha)}\right), \alpha=0,1,2, \ldots$, that have the same solution space as $\mathcal{R}$ and which satisfy the local parametric $h$-principle for $\alpha>\alpha_{0}$, where $\alpha_{0}$ is some positive integer.

To elaborate this let $E \longrightarrow M$ be a $C^{\infty}$-fibration and $G \longrightarrow M$ be a $C^{\infty}$ vector bundle over a manifold $M$. We denote by $\mathcal{E}^{\alpha}$ and $\mathcal{G}^{\alpha}$, respectively, the spaces of $C^{\alpha}$ sections of $E$ and $G$ with the fine $C^{\alpha}$ topologies, for $\alpha=$ $1,2, \ldots, \infty$. Let $\mathcal{D}: \mathcal{E}^{r} \longrightarrow \mathcal{G}^{0}$ be a $C^{\infty}$ differential operator of order $r$, which means that $\mathcal{D}$ is given by a $C^{\infty}$ bundle map $\Delta: E^{(r)} \rightarrow G$ such that $\mathcal{D}(f)=\Delta \circ j_{f}^{r}$. As a consequence, we obtain a sequence of bundle maps $\Delta_{\alpha}: E^{(r+\alpha)} \rightarrow G^{(\alpha)}$ such that $j_{\mathcal{D}(f)}^{\alpha}=\Delta_{\alpha} \circ j_{f}^{r+\alpha}$, where $\alpha$ is any non-negative integer.

Let $V$ denote the subbundle of $T E$ consisting of all vectors which are tangent to the fibres of $E$ over points of $M$. We shall refer $V$ as the vertical tangent bundle of $E$. For any section $f$ of $E$, the vector space of $C^{\beta}$ sections of the pullback bundle $f^{*} V$ will be denoted by $\mathcal{E}_{f}^{\beta}$. The space $\mathcal{E}_{f}^{\beta}$ is defined as the infinite-dimensional tangent space of $\mathcal{E}$ at $f$. It is not difficult to see that when $E$ is a vector bundle, $f^{*} V$ is canonically isomorphic to $E$ and therefore $\mathcal{E}_{f}^{\beta}$ is isomorphic to $\mathcal{E}^{\beta}$.

The linearization $L_{f}$ of $\mathcal{D}$ at $f$ is a map $L_{f}: \mathcal{E}_{f}^{r} \longrightarrow \mathcal{G}^{0}$ which is defined as follows:

$$
L_{f}(y)=\left.\lim _{t \rightarrow \infty} \frac{\partial}{\partial t} \mathcal{D}\left(f_{t}\right)\right|_{t=0}
$$

where $f_{t}$ is a differentiable curve in $\mathcal{E}^{r}$ such that $f_{0}=f$ and the tangent to $f_{t}$ at $t=0$ is $y \in \mathcal{E}_{f}^{r}$. Clearly, $L_{f}$ is a linear differential operator of order $r$ in $y$ and $L(f, y)=L_{f}(y)$ is a differential operator of order $r$ in both $f$ and $y$.

Let $A \subset E^{(d)}$ be an open relation of order $d$ for some $d \geq r$, and $\mathcal{A}$ denote the space of solutions of the relation $A$. Clearly, $\mathcal{A}$ is contained in $\mathcal{E}^{d}$, and $\mathcal{A}^{\alpha+d}=\mathcal{A} \cap \mathcal{E}^{\alpha+d}$ is an open subset of $\mathcal{E}^{\alpha+d}$ in the fine $C^{\alpha+d}$ topology. A solution of $A$ will be referred as an $A$-regular section of $E$.

$\mathcal{D}$ is said to be infinitesimally invertible over the subset $\mathcal{A} \subset \mathcal{E}^{d}$ if for every $f \in \mathcal{A}$ there is a linear differential operator $M_{f}: \mathcal{G}^{s} \longrightarrow \mathcal{E}_{f}^{0}$ of a certain order $s$ (independent of $f$ ) such that the following properties are satisfied: 
(1) The global operator

$$
M: \mathcal{A}^{d} \times \mathcal{G}^{s} \longrightarrow T\left(\mathcal{E}^{0}\right)
$$

is a differential operator that is given by a $C^{\infty}$ map $A \oplus G^{(s)} \longrightarrow V$.

(2) $L(f, M(f, g))=g$ for all $f \in \mathcal{A}^{d+r}$ and $g \in \mathcal{G}^{r+s}$, where $M(f, g)=$ $M_{f}(g)$. In other words, $M_{f}$ is a right inverse of $L_{f}$.

The integer $d$ is called the defect of the infinitesimal inversion $M[8,2.3 .1]$.

We now quote two results from $[\mathbf{8}]$ which are consequences of an Implicit Function Theorem (due to Gromov) in the context of differential operators.

Theorem A.2 $\left(\left[\mathbf{8}, 2.3 .2(\mathrm{~B}),\left(\mathrm{D}^{\prime \prime}\right)\right]\right)$. Suppose that $\mathcal{D}$ is a $C^{\infty}$ differential operator of order $r$ and it admits an infinitesimal inversion of defect $d$ on $\mathcal{A}$.

(i) The operator $\mathcal{D}: \mathcal{A}^{\infty} \longrightarrow \mathcal{G}^{\infty}$ is an open map in the respective fine $C^{\infty}$ topologies.

(ii) The sheaf of $A$-regular solutions of the differential equation $\mathcal{D}=g$ is microflexible, where $g$ is a smooth section of $G$.

Definition A.6. A local section $f$ of $E$, defined on a neighbourhood of some $x \in M$, is said to be an infinitesimal solution of $\mathcal{D}=g$ of order $\alpha$ if the $\alpha$-jet of $\mathcal{D}(f)-g$ is zero at $x$.

Let $\mathcal{R}^{\alpha} \subset E^{(\alpha+r)}$ consist of $(\alpha+r)$ - jets of infinitesimal solutions of $\mathcal{D}=g$ of order $\alpha$ and let $\mathcal{R}^{0}$ be denoted as $\mathcal{R}$. Since $j_{\mathcal{D}(f)}^{\alpha}=\Delta_{\alpha} \circ j_{f}^{r+\alpha}$, therefore, $\mathcal{R}^{\alpha}=\left(\Delta_{\alpha}\right)^{-1}\left(j_{g}^{\alpha}\right)$.

Define

$$
\mathcal{R}_{\alpha}=\mathcal{R}^{\alpha} \cap\left(p_{d}^{\alpha+r}\right)^{-1}(A),
$$

where $p_{d}^{\alpha+r}: E^{(\alpha+r)} \longrightarrow E^{(d)}$ is the canonical projection map for $\alpha \geq d-r$. The relations $\mathcal{R}_{\alpha}$ have the same $C^{\infty}$ solutions for all $\alpha \geq d-r$, namely the $C^{\infty}$ solutions of the equation $\mathcal{D}(x)=g$ in $\mathcal{A}$.

Let $\Phi_{\text {reg }}$ denote the sheaf of $A$-regular solutions of the equation $\mathcal{D}=g$ with the $C^{\infty}$ compact open topology and let $\Psi_{\alpha}$ be the sheaf of sections of $\mathcal{R}_{\alpha}$ with $C^{0}$ compact open topology.

Proposition A.1 ([8, 2.3.2( $\left.\left.\left(\mathrm{D}^{\prime}\right),\left(\mathrm{D}^{\prime \prime}\right)\right]\right)$. If $\mathcal{D}$ admits an infinitesimal inversion of order $s$ and defect $d$ on $\mathcal{A}$ then the map $J: \Phi_{\text {reg }} \longrightarrow \Psi_{\alpha}$, defined by $J(\phi)=j_{\phi}^{r+\alpha}$, is a local weak homotopy equivalence for each $\alpha \geq \max (d+s, 2 r+2 s)$. In other words, $\mathcal{R}_{\alpha}$ satisfies the local parametric h-principle.

Thus we see that there is a large class of relations $\mathcal{R}$ which satisfy the local $h$-principle and for which the solution sheaves are microflexible. The following result of Gromov in $\left[\mathbf{8}, 2.2 .3\left(\mathrm{C}^{\prime}\right)\right]$ is the central result in the theory of $h$-principle as far as the sheaf technique is concerned: 
Theorem A.3. If $\Phi$ is a microflexible sheaf on a manifold $M$ and $N$ is an embedded submanifold of positive codimension, then $\Phi \mid N$ is flexible, provided there is a class of 'acting diffeotopies' $\mathcal{D}_{0}$ which 'sharply moves $N$ '.

We now explain the notion of 'acting diffeotopies' and 'sharply moving diffeotopies'.

Action of diffeotopies: Let $\Phi$ be a topological sheaf over a manifold $M$ and $U^{\prime}$ an open subset of $M$. Consider a diffeotopy $\delta_{t}: U \rightarrow U^{\prime}$ that moves an open subset $U \subset U^{\prime}$ inside $U^{\prime}$, where $\delta_{0}=$ id. Let $\Phi^{\prime}$ be a subset of $\Phi\left(U^{\prime}\right)$, and the diffeotopy $\delta_{t}$ act on $\Phi^{\prime}$ by assigning a homotopy of sections $\delta_{t}^{*} \phi$ in $\Phi(U)$ to every $\phi \in \Phi^{\prime}$ such that $\delta_{0}^{*} \phi=\left.\phi\right|_{U}$ and the following conditions are satisfied:

(1) If two sections $\phi_{1}$ and $\phi_{2}$ in $\Phi^{\prime}$ are such that $\phi_{1}\left(u^{\prime}\right)=\phi_{2}\left(u^{\prime}\right)$ for some $u^{\prime} \in U^{\prime}$ and if $\delta_{t_{0}}(u)=u^{\prime}$ for some $u \in U$, then $\delta_{t_{0}}^{*} \phi_{1}(u)=\delta_{t_{0}}^{*} \phi_{1}(u)$. In particular, if the two sections $\phi_{1}$ and $\phi_{2}$ restrict to the same section on $U$, then $\left(\left.\delta_{t}\right|_{U}\right)^{*} \phi_{1}=\left(\left.\delta_{t}\right|_{U}\right)^{*} \phi_{2}$.

(2) If $U_{0}$ is a maximal open subset where $\delta_{t}$ is constant, (that is, $\delta_{t}(x)=x$ for all $x \in U_{0}$, ) then $\delta_{t}^{*} \phi$ is also constant on $U_{0}$ (that is $\delta_{t}^{*} \phi=\phi$ on $\left.U_{0}\right)$.

(3) If the diffeotopy $\delta_{t}$ is constant for $t \geq t_{0}$, then $\delta_{t}^{*} \phi$ is also constant for $t \geq t_{0}$ for some $t_{0} \in[0,1]$.

(4) If $\phi_{p} \in \Phi^{\prime}, p \in P$, is a continuous family of sections then the family $\delta_{t}^{*} \phi_{p}$ is jointly continuous in $p$ and $t$.

Conditions (2) and (3) are natural in the sense that they make the action compatible with the presheaf structure.

One must note that this is a partial action as $\delta_{t}$ need not in general act on $\Phi(U)$. Further, there is no condition on the subset $\Phi^{\prime}$.

Example A.1. Let $\Phi$ denote the sheaf of sections of the product bundle $M \times N$ over $M$. Then $\operatorname{Diff}(M)$, the pseudogroup of local diffeomorphisms of $M$, has a natural action on $\Phi$ given by $\delta \cdot \bar{f}=\bar{\delta}^{-1} \bar{f} \delta$, where $\bar{f} \in \Phi$ and $\bar{\delta}: U \times N \rightarrow V \times N$ is given by $\bar{\delta}(x, y)=(\delta(x), y)$. We can extend this action to an action by diffeotopies of $M$. However, if we consider the subsheaf $\Phi_{Y}$ of sections of $M \times N$ whose images lie in an open subset $Y$ then $\Phi_{Y}$ is not invariant under this action. In this case we get only a partial action by diffeotopies: indeed, if $\delta_{t}$ is sufficiently $C^{0}$-close to the identity map then it acts on $\Phi_{Y}(U)$. More generally, if $\delta_{t}$ is a diffeotopy that moves $U$ in $M$ and if there is an open subset $Y^{\prime} \subset(U \times N)$ such that $\bar{\delta}_{t}\left(Y^{\prime}\right) \subset Y$ for all $t \in[0,1]$, then $\delta_{t}$ acts on the sheaf $\Phi_{Y}$.

Definition A.7. We fix a metric $d$ on $M$. Let $M_{0}$ be a submanifold of $M$ of positive codimension which lies in an open subset $U^{\prime}$ of $M$. A class of 
diffeotopies $\mathcal{D}$ on $M$ is said to sharply move $M_{0}$ in $M$ if given any hypersurface $S$ in $M_{0}$ and any positive numbers $\varepsilon$, we can obtain a diffeotopy $\delta_{t}: \mathrm{Op} M_{0} \rightarrow U^{\prime}$ in $\mathcal{D}$ which satisfies the following conditions:

(1) $\delta_{0}$ is the identity map;

(2) $\left.\delta_{t}\right|_{O p v}$ is identity for all $v \in M_{0}$ for which $d(v, S) \geq \varepsilon$;

(3) $d\left(\delta_{1}(S), M_{0}\right)>r$ for some positive number $r$.

We end this section with the following result of $h$-principle.

Theorem A.4. Let $M=M_{0} \times \mathbb{R}$. Suppose that $\mathcal{R}$ satisfies the local parametric h-principle and the solution sheaf $\Phi$ of $\mathcal{R}$ is microflexible. If the fibre-preserving diffeotopies of $M$ act on $\Phi$ then $\mathcal{R}$ satisfies the $h$-principle.

Proof. Since the fibre-preserving diffeotopies of $M$ sharply move the submanifold $M_{0}$, it follows from Theorem A.3 that a section of $\mathcal{R}$ can be homotoped to a holonomic section $j_{f}^{r}$ over an open neighbourhood $U$ of $M_{0} \times\{0\}$ in $M$. Since $M$ is split as $M_{0} \times \mathbb{R}$, we can deform $M$ into $U$ by a smooth one-parameter family of embeddings $F_{t}: M_{0} \times \mathbb{R} \longrightarrow M_{0} \times \mathbb{R}, 0 \leq$ $t \leq 1$, such that $F_{t}$ is fibre-preserving and $F_{1}$ takes $M$ into $U$. Since $F_{t}$ acts on $\Phi, F_{1}^{*} f$ is a global solution of $\mathcal{R}$. This proves the theorem.

\section{References}

[1] G. D'Ambra, Nash $C^{1}$-embedding theorem for Carnot-Carathodory metrics, Differential Geom. Appl. 5(2), (1995), 105-119.

[2] G. D'Ambra and M. Datta, Isometric $C^{1}$-immersions for pairs of Riemannian metrics, Asian J. Math. 6(2), (2002), 373-384.

[3] G. D'Ambra and M. Datta, Lipschitz solutions to the isometry relation for pairs of Riemannian metrics, Pacific J. Math. 237(2), (2008), 223-240.

[4] G. D'Ambra and A. Loi, A symplectic version of Nash $C^{1}$-isometric embedding theorem, Differential Geom. Appl. 16(2), (2002), 167-179.

[5] M. Datta and R. Islam, Submersion on open symplectic manifolds, Topol. Appl. 156(10), (2009), 1801-1806.

[6] Y. Eliashberg and N. Mishachev, Introduction to the h-principle, Graduate Studies in Math. 48, Amer. Math. Soc., Providence, RI, 2002.

[7] M. Golubitsky and V. Guillemin, Stable mappings and their singularities, Graduate Texts in Math. 14, Springer-Verlag, Berline, 1973.

[8] M. Gromov, Partial differential relations, Ergebnisse der Mathematik und ihrer Grenzgebiete 3, Folge Band 9, Springer-Verlag, Berlin, 1986.

[9] D. McDuff and D. Salamon, Introduction to symplectic topology, Oxford Math. Monographs, The Clarendon Press, Oxford, 1995.

[10] J. Milnor, Lectures on the h-cobordism theorem, Princeton University Press, Princeton, NJ, 1965.

[11] Nash, J. $C^{1}$-isometric embeddings, Ann. Math. 60 (1954), 383-396. 
Statistics and Mathematics Unit

Indian Statistical Institute

203, B.T. ROAD,

Calcutta 700108,

INDIA

E-mail address: mahuyad@gmail.com

Received 08/14/2009, accepted 06/25/2010 
